\title{
Transcatheter Interatrial Shunts for the Treatment of Heart Failure with Preserved Ejection Fraction
}

\author{
Anju Bhardwaj, ${ }^{\circledR}$ Vishal Y. Parikh, ${ }^{[0}$ Ajith Nair ${ }^{(0)}$ \\ University of Texas Health Science Center at Houston, ${ }^{1}$ Texas - EUA \\ Rochester Regional Health, ${ }^{2}$ Nova York - EUA \\ Baylor College of Medicine, ${ }^{3}$ Texas - EUA
}

\begin{abstract}
Heart failure with preserved ejection fraction (HFpEF) is a clinical syndrome, which accounts for about $50 \%$ of patients with heart failure (HF). The morbidity and mortality associated with HFpEF is similar to HFrEF. Clinical trials to date have failed to show a benefit of medical therapy for HFpEF, which may due to lack of uniform phenotypes and heterogeneous population. In addition, medical therapy proven for HFrEF may not address the pathophysiologic basis for HFpEF. Left atrial remodeling and dysfunction is central to HFpEF and accounts for secondary pulmonary hypertension and pulmonary vascular congestion that frequently occurs with exertion. Interatrial shunts represent a novel treatment modality for HFpEF. These shunts allow for left atrial decongestion and a reduction in pulmonary venous hypertension during exercise leading to improvements in hemodynamics, functional status and quality of life. Trials to date have demonstrated safety and short-term efficacy of these devices for HFpEF. The long-term benefits are currently being evaluated in ongoing trials. If effective, the use of interatrial shunts may be a new therapeutic paradigm for the treatment of HFpEF.
\end{abstract}

\section{Introduction}

Heart failure with preserved ejection fraction (HFpEF) is a clinical syndrome, which accounts for

\section{Keywords}

Heart Failure; Stroke Volume; Hypertrophy, Left Ventricular; Heart Atria; Transcatheter. about $50 \%$ of patients with heart failure (HF), with an estimated prevalence exceeding 5 million people. ${ }^{1,2}$ Not only is it associated with poor quality of life, but the mortality and morbidity associated with HFpEF are also similar to those reported for heart failure with reduced ejection fraction (HFrEF). ${ }^{3}$ While the management of HFrEF has improved significantly over the last two decades, little or no progress has been made regarding optimal and effective treatment for HFpEF. This lack of evidence-based clinical strategies for the treatment of HFpEF may be due to the diverse phenotypes of HFpEF. ${ }^{4}$ While medical therapy has failed to yield clear benefits, there has been interest in device-based therapies, including the creation of interatrial shunts to "unload" the left atrium. This review discusses the diagnosis and pathogenesis of HFpEF, the rationale behind interatrial shunts (IAS) and the current IAS devices under clinical evaluation.

Diagnosis of Heart failure with preserved ejection fraction

Given that HFpEF has several distinct phenotypes and pathophysiological mechanisms, its diagnosis is challenging secondary to the lack of a uniform diagnostic algorithm. Moreover, it is diagnosed primarily by excluding other potential noncardiac causes of symptoms suggestive of HF. The diagnosis does not depend on a single criterion. Furthermore, non-invasive parameters are not consistently reliable for its diagnosis, as abnormalities in diastology may only be revealed on exertion. The current criteria proposed to define HFpEF include: a) clinical signs

Mailing Address: Ajith Nair

7200 Cambridge St. Ste 6C - Houston

E-mail: ajith.nair@bcm.edu 
or symptoms of $\mathrm{HF}$; b) evidence of preserved or normal left ventricular ejection fraction (LVEF); and c) evidence of abnormal left ventricular (LV) diastolic dysfunction that can be determined by Doppler echocardiography or cardiac catheterization. ${ }^{5}$ Other diagnostic parameters include: left ventricular hypertrophy $(\mathrm{LVH})$, left atrial enlargement, elevated serum natriuretic peptide (NP) levels and history of atrial fibrillation (AF). ${ }^{6}$ It is well established that normal NP levels have a very high negative predictive value for excluding HF, but normal NP levels do not exclude HFpEF due to other confounding variables. ${ }^{7-9}$ Reddy et al., ${ }^{10}$ have retrospectively derived a composite HFpEF diagnostic score (Table 1), which includes clinical characteristics (age $>60$ years, obesity, atrial fibrillation, treatment with $\geq 2$ antihypertensive drugs) and echocardiographic measurements $\left[\mathrm{E} / \mathrm{e}^{\prime}>9\right.$, pulmonary artery systolic pressure (PASP) $>35 \mathrm{mmHg}] .{ }^{10}$ However, the gold standard test for confirming HFpEF is invasive right heart catheterization with elevated left ventricular filling pressures: an elevated pulmonary capillary wedge pressure (PCWP) $\geq 15 \mathrm{mmHg}$ at rest or $\geq 25 \mathrm{mmHg}$ during exercise measured by right catheterization. ${ }^{5}$

\section{Pathophysiology of Heart Failure with Preserved Ejection Fraction}

HFpEF is a complex entity with many traditional and nontraditional cardiovascular risk factors, such as hypertension (HTN), diabetes mellitus (DM), obesity, renal or pulmonary disease, influencing its pathophysiology. ${ }^{11}$ These comorbidities, particularly obesity, induce a systemic proinflammatory state evident from increased circulation of interleukin-6 and tumor necrosis factor alpha (TNF- $\alpha$ ). This induces microvascular endothelial cells to produce reactive oxygen species, thus limiting the availability of nitric oxide (NO) for cardiomyocytes. This leads to decreased cGMP production, which decreases protein kinase $G$ and hypophosphorylates the protein titin, thereby inducing concentric left ventricular hypertrophy and stiffness of cardiomyocytes, respectively. This myocardial stiffness and fibrosis cause diastolic myocardial dysfunction, which is the hallmark of HFpEF. ${ }^{12}$ Other pathophysiological processes include: increased systemic vascular resistance and arterial stiffness, abnormal ventricular arterial coupling, ventricular dyssynchrony, atrial dysfunction, impaired right ventricular function, pulmonary hypertension, coronary microvascular dysfunction, and chronotropic incompetence. ${ }^{13} \mathrm{HFpEF}$ is typically characterized by decreased stroke volume and cardiac output. This impaired LV diastolic relaxation leads to elevated left atrial pressure during physical activity, which is deemed the key driver of symptoms in HFpEF. ${ }^{14}$

\section{Left Atrial Remodeling}

The decrease in left atrial (LA) function and remodeling is a central pathophysiological phenomenon in HFpEF. Pressure and volume overload can cause left atrial dysfunction at a cellular level. LA remodeling is thought to be due to mechanical stretch, multiple cytokine activation, atrial fibrosis, and cellular apoptosis. ${ }^{15}$ Typically in HFpEF, compared to HFrEF, there are lower LA volumes, higher LA

Table 1: Heart Failure with Preserved Ejection Fraction (HFpEF) - Diagnosis

\begin{tabular}{|c|c|c|c|}
\hline & Clinical Variable & Values & Points \\
\hline \multirow{2}{*}{$\mathbf{H}$} & Heavy & Body Mass Index $>30 \mathrm{~kg} / \mathrm{m} 2$ & 2 \\
\hline & Hypertension & Two or more hypertensive medications & 1 \\
\hline $\mathbf{F}$ & Atrial Fibrillation & Paroxysmal or Persistent & 3 \\
\hline $\mathbf{P}$ & Pulmonary Hypertension & Doppler echocardiographic estimated PA pressures $>35 \mathrm{mmHg}$ & 1 \\
\hline E & Elder & Age $>60$ years & 1 \\
\hline $\mathbf{F}$ & Filling Pressures & Doppler Echo E/e' $>9$ & 1 \\
\hline \multicolumn{2}{|c|}{ H2FPEF Score } & & Sum 0-9 \\
\hline
\end{tabular}


peak pressures, stiffness, pulsatility, and stress wall variations. High LA stiffness also plays a significant role in increased atrial fibrillation burden. There is also a relationship between LA dysfunction and pulmonary vascular disease, which contributes to right heart failure, most likely due to reduced pulmonary arterial compliance. This decreased compliance can lead to RV to PA uncoupling, which is unmasked with exercise and prevalent in more advanced stages of HFpEF. LA dysfunction in HFpEF is also correlated with mortality among this population. ${ }^{16}$

The "stiff left atrial syndrome" is marked by a noncompliant left atrium and can be seen after cardiac surgeries or after catheter ablation for atrial fibrillation. These patients can develop left atrial diastolic dysfunction and associated pulmonary hypertension. Gibson et al., ${ }^{17}$ demonstrated that severe LA scarring, small LA, diabetes mellitus, OSA, and high LA pressures were predictive of the development of this syndrome in atrial fibrillation patients who underwent catheter ablation. ${ }^{17}$ Interestingly, these clinical variables are commonly seen in patients with HFpEF. Case reports document the development of stiff left atrial syndrome after atrial fibrillation ablations, marked by a rise in wedge pressures and prominent $\mathrm{v}$ waves during right heart catheterization with exercise. ${ }^{18}$ Acute pulmonary vascular congestion may result from an abrupt rise in left atrial pressures. ${ }^{19}$ Atrial septostomy can significantly improve symptoms, increase peak $\mathrm{VO} 2$, and mitigate $\mathrm{RV}$ dysfunction and pulmonary hypertension. ${ }^{20}$ Interatrial shunt devices may promote reverse LA remodeling by decreasing pressure and volume overload, thereby decreasing the risk of RV failure and improving mortality in patients with HFpEF.

\section{Failure of Medical Therapy for HFPEF}

To date, the pharmacological interventions approved for HFrEF have not shown to improve any outcomes in HFpEF. ${ }^{21}$ Angiotensin receptor blockers failed to demonstrate benefit in the CHARM-Preserved trial. ${ }^{22}$ Spironolactone did not meet its primary endpoint of death from cardiovascular causes, aborted cardiac arrest, or heart failure hospitalizations in the TOPCAT trial. ${ }^{23}$ Inconsistent patient selection and medication administration may have undermined the trial. ${ }^{24}$ The PARAGON-HF trial, which compared sacubitrilvalsartan with valsartan alone in patients with HFpEF, also failed to meet its primary endpoint of lower heart failure hospitalization rates or cardiovascular death among patients with heart failure and an ejection fraction of $45 \%$ or higher. ${ }^{25}$ However, there was a suggestion that women may have benefited from sacubitril-valsartan more than men in reducing heart failure hospitalization. ${ }^{26}$

\section{Interatrial Shunting for Heart Failure with Preserved Ejection Fraction}

It has been observed that, compared to patients with isolated mitral stenosis, patients with Lutembacher's syndrome (mitral stenosis and congenital atrial septal defect) have less profound symptoms due to LA pressure offloading. In similar contexts, patients with HFpEF have higher filling pressures (at rest and/ or with exercise), which drives fluid retention and acute decompensations; ${ }^{27,28}$ interatrial shunt devices have been used to reduce left atrial pressure by allowing modest left to right shunting in HFpEF. ${ }^{29,30}$ Interatrial pressure gradient regulates flow through the interatrial shunt to relieve left atrial pressure overload. In a cardiovascular simulation model, Kaye et al., ${ }^{30}$ demonstrated that a shunt diameter of $8-9 \mathrm{~mm}$ lowered wedge pressure without an increase in right atrial or pulmonary artery. ${ }^{30}$ The target patient population for such treatment included HF patients with a high-pressure gradient between the left and right atrium without RV dysfunction. Patients with RV dysfunction are not candidates for shunts due to concerns for RV overload and progressive RV failure.

\section{Devices (Table 2)}

The Interatrial shunt device (IASD, Corvia Medical) consists of a $19 \mathrm{~mm}$ wide nitinol mesh with multiple legs, radiopaque markers and an 8 $\mathrm{mm}$ central communication to create the interatrial septal defect. ${ }^{31,32}$ The legs of this device are flat on the LA side to minimize the risk of thrombus formation. Dual antiplatelet therapy with aspirin and clopidogrel is recommended for 6 months, followed by lifelong aspirin monotherapy. Patients taking oral anticoagulants are recommended to continue their existing oral anticoagulant therapy after the procedure with endocarditis prophylaxis advised for a minimum of six months after the implantation. ${ }^{32}$

The V-wave device (V-wave Inc) is a tri-leaflet porcine tissue valve on an hourglass shaped device also made of nitinol, encapsulated by expanded 
Table 2 - Interatrial Septal Devices

\begin{tabular}{|c|c|c|c|}
\hline Device & Characteristics & Studies & Notes \\
\hline & & REDUCE LAP- HF and & \multirow{6}{*}{$\begin{array}{l}\text { REDUCE LAP-HF II: Phase III } \\
\text { RCT that is currently enrolling. }\end{array}$} \\
\hline IASD $®$ system & & REDUCE LAP-HF I study & \\
\hline \multirow{4}{*}{$\begin{array}{l}\text { (Corvia Medical Inc., } \\
\text { Tewkesbury, Massachusetts) }\end{array}$} & $8 \mathrm{~mm}$ ASD & (phase II trial). & \\
\hline & Shunt fraction 1.2-1.3 & Evidence of safety of the IASD & \\
\hline & & with no differences between & \\
\hline & & MACCRE in IASD vs sham. & \\
\hline \multirow{2}{*}{$\begin{array}{l}\text { V-wave Shunt } \\
\text { (V-Wave Ltd., Caesarea, Israel) }\end{array}$} & \multirow{2}{*}{$\begin{array}{l}5 \mathrm{~mm} \text { unidirectional } \\
\text { Nitinol }\end{array}$} & Initial safety & \multirow{2}{*}{$\begin{array}{l}\text { RELIEVE-HF. V Wave to be } \\
\text { assessed in both HFrEF and } \\
\text { HFpEF }\end{array}$} \\
\hline & & $\begin{array}{l}\text { and hemodynamic benefits in } \\
\text { HFrEF and HFpEF }\end{array}$ & \\
\hline \multirow{2}{*}{ Atrial Flow Regulator } & \multirow{4}{*}{$\begin{array}{l}\text { 2, 5and } 10 \mathrm{~mm} \\
4-10 \mathrm{~mm}\end{array}$} & Initial improvement in & \\
\hline & & symptoms, functional class, & \\
\hline \multirow{2}{*}{ (Occlutech, Istanbul, Turkey) } & & hemodynamic sand biomarkers & \\
\hline & & in HFrEF and HFpEF. & \\
\hline
\end{tabular}

polytetrafluoroethylene on the left atrial side with the three porcine pericardial leaflets sutured with Prolene to ensure a $5 \mathrm{~mm}$ unidirectional (left to right) shunt. ${ }^{33,34}$ The PTFE is designed to improve blood flow and restrict new growth over the device. Following implantation, patients require anticoagulation with warfarin or direct-acting oral anticoagulant (DOAC) for three months and low-dose aspirin indefinitely. ${ }^{35}$

The Atrial Flow Regulator (AFR, Occlutech) is another device made of nitinol mesh and a central hole. There are three waist sizes to suit the atrial septal thickness $(2,5$ and $10 \mathrm{~mm})$ and the fenestration diameter varies from 4-10 mm, which was tested in a small pilot study in patients with HFrEF and HFpEF. ${ }^{31,34,36}$

\section{Implantation Technique}

For most interatrial devices, implantation is performed under general anesthesia, using transfemoral approach with fluoroscopic and transesophageal or intracardiac echocardiographic (ICE) guidance. Following a transseptal puncture, a 14-16 Fr sheath is advanced into the LA, and each device is deployed with its respective delivery system with balloon pre-dilation recommended for implantation of the AFR device. ${ }^{36}$ The left side of the device is initially opened, with the entire system pulled back, ensuring tenting at IAS, followed by deployment of the right side of the device. (Figure 1) The delivery system and guide wire are then removed, and hemostasis is achieved.

\section{Clinical Evidence}

The first human experience of IASD (Corvia Medical) was a non-randomized, pilot study by Sondergaard et al., ${ }^{32}$ in 11 patients with LVEF $>45 \%$, baseline PCWP $>15 \mathrm{~mm} \mathrm{Hg}$ at rest or $>25 \mathrm{~mm} \mathrm{Hg}$ during exercise, $\geq$ one hospitalization for heart failure within the past 12 months, or persistent NYHA class III /ambulatory class IV for at least 3 months. ${ }^{32}$ The average age of these patients was 71 years, with an average left ventricular ejection fraction (LVEF) of 57\%, average PCWP of 19 $\mathrm{mm} \mathrm{Hg}$, and median NT-proBNP of $148 \mathrm{pg} / \mathrm{mL}$. Devices were successfully implanted in all but one patient, in whom the insertion of a new device corrected device malposition. There were no device-related complications, such as migration or loss of patency. After 30 days, LV filling pressures were significantly reduced by $5.5 \mathrm{mmHg}$ (28\%, $19.7 \pm 3.4$ vs. $14.2 \pm 2.7 ; \mathrm{P}=0.005)$, and there were significant improvements in 6MWT distance, quality of life, and NYHA class, with no changes in PAP or RAP. At one year all patients survived and the symptomatic improvement (as measured by NYHA class) was sustained, although some patients required an increase in their daily dose of loop diuretics.

The Reduce Elevated Left Atrial Pressure in Patients 


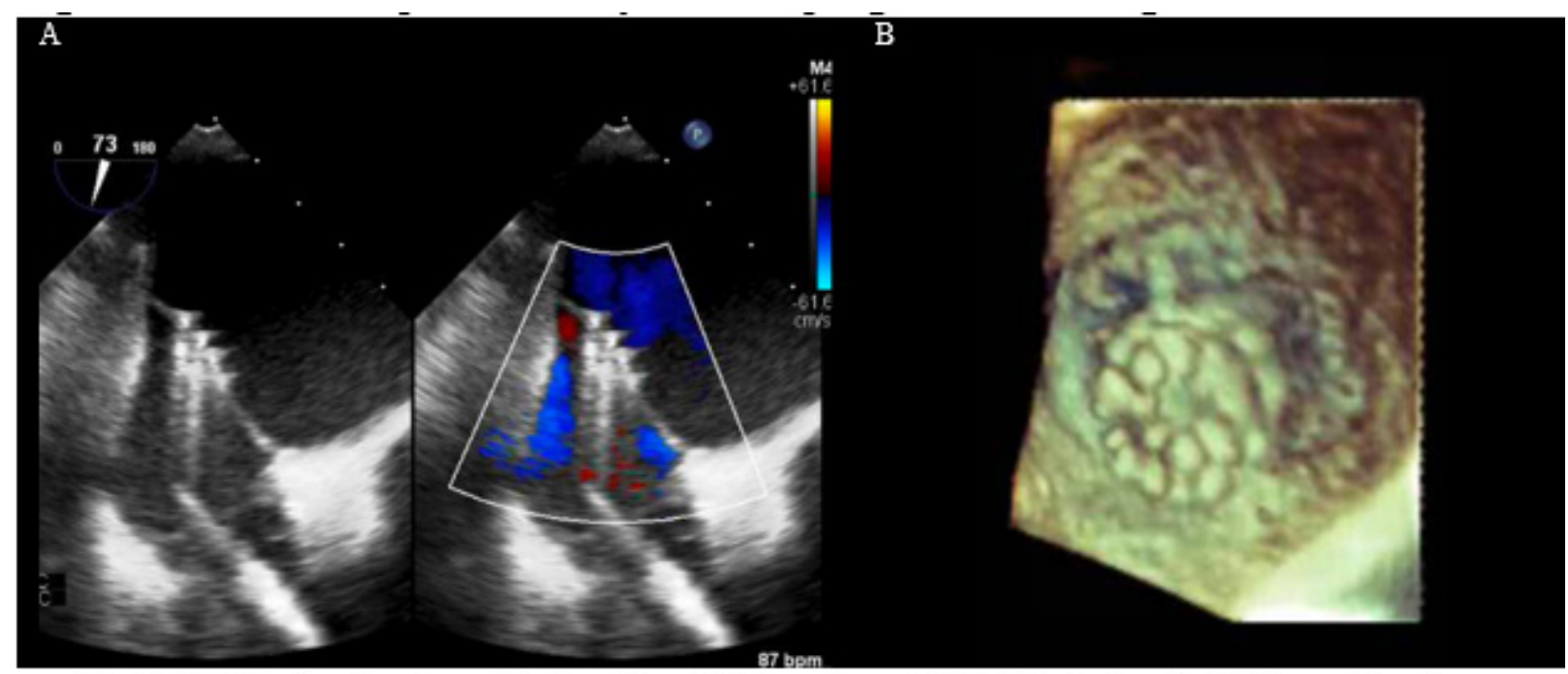

\section{A. IASD device deployed in the interatrial septum. B. IASD device in 3-dimensional imaging.}

Figure 1 - Intra-atrial Septal Device by Transesophageal Echocardiogram

with HF (REDUCE LAP-HF) study was a multicenter, prospective, non-randomized, open-label, single-arm study in patients with symptomatic HFpEF (NYHA class II-IV) and elevated PCWP (>15 mm Hg at rest and $>25 \mathrm{~mm} \mathrm{Hg}$ during exercise). ${ }^{29,37} \mathrm{~A}$ total of 68 patients with symptomatic HFpEF underwent an IASD system II device implantation. The average age of subjects was 69 years, average LVEF of $47 \%$, mean PCWP at rest was $17 \mathrm{~mm} \mathrm{Hg}$ and median NTproBNP was $377 \mathrm{pg} /$ $\mathrm{mL}$. Overall, the device was successfully implanted in 64 patients. At a six-month follow-up, there was no significant change in PCWP at rest, but a significant drop in PCWP upon exertion was observed. Sustained device patency at 6 months was confirmed by left-toright shunting. Furthermore, IASD was associated with significant improvements in symptoms, quality of life, and functional status at six months, which were sustained at the 12-month follow-up. ${ }^{29,38}$ Kaye et al., ${ }^{39}$ investigated the impact of IASD on HFpEF mortality over a follow up of 739 days. The observed mortality rate of the IASDtreated cohort was 3.4/100 patient-year, representing a $33 \%$ reduction in all-cause mortality rates $(p=0.02) .{ }^{39}$

These observations were further validated in a sham-controlled randomized trial, REDUCE LAPHF I study (Reduce Elevated Left Atrial Pressure in Patients With Heart Failure), which was a phase 2, randomized, parallel-group, blinded multicenter trial in patients with NYHA class III or ambulatory class IV HF. Enrolled patients had an EF $\geq 40 \%$, exercise PCWP $\geq 25 \mathrm{~mm} \mathrm{Hg}$, and PCWP-right atrial pressure gradient $\geq 5 \mathrm{~mm} \mathrm{Hg}$. Forty-four patients were randomized (1:1) to the IASD system II device placement versus a sham procedure (femoral venous access with intracardiac echocardiography, but no IASD placement). ${ }^{40}$ Mean age of participants was $70 \pm 9$ years, and $50 \%$ were female. At one-month follow-up, the IASD group resulted in greater reduction in PCWP compared with sham control ( $\mathrm{P}=0.028$ accounting for all stages of exercise). Peak PCWP decreased by $3.5 \pm 6.4 \mathrm{~mm} \mathrm{Hg}$ in the treatment group versus $0.5 \pm 5.0 \mathrm{~mm} \mathrm{Hg}$ in the control group $(\mathrm{P}=0.14)$. There were no major periprocedural complications or adverse cardiac, cerebrovascular, or renal events reported in the IASD group at one month. At one-year follow-up, there was $100 \%$ device patency. There were no statistically significant differences in functional class, exercise capacity, HF hospitalization or diuretic use between the two groups at one year, although this may be attributed to the limited sample size $(\mathrm{n}=44) .{ }^{41}$ Despite an increase in right ventricular size, the IASD was not associated with right ventricular dysfunction, and improvements were observed in pulmonary vascular function at rest and during exercise. ${ }^{42}$ 
The V-wave device was the first IAS device implanted in a patient with HFrEF. ${ }^{35}$ Initial experience with this device was reported by Del Trigo et al., ${ }^{33}$ in 10 patients with HFrEF. ${ }^{33}$ A single-arm multicenter assessment in 38 patients (30 with HFrEF and 8 with HFpEF) demonstrated no periprocedural mortality, although pericardial tamponade occurred in one patient $(2.6 \%) .{ }^{43}$ After 3 and 12 months, improvements in NYHA functional class, QoL, and 6MWT were observed, although there was no significant change in hemodynamic parameters at 12 months. After an extended follow-up of 28 months, ten deaths were reported (of which eight were from cardiovascular causes), and two patients required advanced therapies (one received an LVAD at 15 months and another received transplant at 27 months). All shunts remained patent at three months but, at one year follow-up, 5 out of 36 (14\%) were occluded, and another 13 (36\%) were stenotic, leading to a shunt stenosis/occlusion rate of $50 \% .{ }^{43} \mathrm{~A}$ comparative analysis between patients with patent and occluded shunts suggested significant improvements in hemodynamic parameters and late clinical outcomes, including death, HF hospitalizations and requirement of advanced therapies in those who maintained patency.

The potential cause of stenosis was deemed to be intra-shunt valve deterioration, which prompted development of a newer generation $\mathrm{V}$-wave device. The second-generation valveless $\mathrm{V}$-wave shunt was studied in ten patients, which remained patent at one-year follow-up. ${ }^{44}$ The efficacy of this device has been currently evaluated in a large randomized trial.

The AFR-PRELIEVE was a prospective, nonrandomized, open-label, multicenter phase 2 pilot study in patients with symptomatic heart failure NYHA Class III /ambulatory class IV and pulmonary capillary wedge pressure (PCWP) $\geq 15 \mathrm{mmHg}$ at rest or $\geq 25 \mathrm{mmHg}$ at exercise, irrespective of LVEF. The study included 36 patients, of whom 20 had HFpEF. Implantation success rate and device patency with the left-to-right shunt was $100 \%$ at the three-month follow-up. ${ }^{36}$ Individual patients from both the HFrEF and HFpEF groups showed improvement in symptoms and surrogate parameters of heart failure, including NYHA class, six-minute walking distance, Kansas City Cardiomyopathy Questionnaire, PCWP and NT-proBNP values. Further evaluation is necessary to determine the long-term benefits of the device in HFPEF.

\section{Future Trials}

The REDUCE LAP-HF TRIAL II (NCT03088033) is a sham-controlled, multicenter, prospective phase 3 trial being conducted to evaluate the clinical efficacy and safety of the IASD II for HFpEF patients with elevated left atrial pressure who remain symptomatic despite appropriate medical management. Patients are randomized to IASD II implantation or to control arm and will be followed for five years for "hard" clinical endpoints (cardiovascular death, non-fatal stroke, need for hospitalization because of acutely decompensated HF). A post-market observational registry is also underway that aims to assess the efficacy and safety of the IASD System II and its benefits on quality of life (REDUCE-LAP HF III, NCT03191656).

Reducing Lung Congestion Symptoms in Advanced Heart Failure (RELIEVE-HF, NCT03499236) is a prospective, multicenter, randomized, double-blinded study which aims at providing data on the safety and clinical effectiveness of the V-Wave Interatrial Shunt System in patients with NYHA functional class III or ambulatory class IV HF, irrespective of LVEF. Approximately 400 patients will be randomized to shunt treatment and a non-implant control arm, and the study will be blinded during follow-up for a minimum of 12 months to a maximum of 24 months. All implanted patients will be followed for five years from the time of the study device implantation.

\section{Limitations of this Modality}

The results of interatrial shunt devices have been encouraging in small studies; however, there remain concerns regarding its efficacy and safety in clinical practice. Even though there was a notable drop in filling pressures, not all studies demonstrated a significant improvement in mortality. Larger clinical trials will address whether pivotal hemodynamic improvements seen in initial studies will translate to benefits in "hard" clinical endpoints and long-term cardiac remodeling.

In addition, long-term adverse outcomes with these devices - including paradoxical embolus, right ventricular dysfunction, tricuspid regurgitation and pulmonary hypertension - will need to be appraised before adoption in standard clinical practice. While the degree of shunt ratios remain small, with Qp:Qs averaging 1:2 to $1: 3$, the impact on right atrial function 
and potential arrhythmias may be of concern ${ }^{45}$ Optimal long-term antiplatelet regimens for these devices will also need to be determined to maintain patency.

\section{Conclusions and Future Perspectives:}

HFpEF carries morbidity and mortality similar to HFrEF, and poses a similar fiscal strain on healthcare. ${ }^{46}$ HFpEF remains a clinically ill-defined entity, given its complex etiology and pathogenesis, and therapeutic options are lacking HFpEF due to the heterogeneity in the patient population. Interatrial shunting appears to be a promising option for treating patients with HFpEF. It may prove to be a safe, cost-effective, and feasible therapy for patients who remain symptomatic due to pulmonary congestion and intrinsically elevated left atrial pressure; it may also be considered as a palliative therapy in selected patients with refractory pulmonary hypertension associated with HFpEF. The current devices seem to be safe in the short and midterm, albeit data on their longterm safety and efficacy are lacking. Ongoing studies that include a more significant number of patients and with extended follow-up periods will determine whether this novel approach is a viable treatment modality for HFPEF.

\section{References}

1. Dunlay SM, Roger VL, Redfield MM. Epidemiology of heart failure with preserved ejection fraction. Nat Rev Cardiol. 2017;14(10):591-602.

2. Go AS, Mozaffarian D, Roger VL, Benjamin EJ, Berry JD, Blaha MJ, et al. Executive summary: heart disease and stroke statistics--2014 update: a report from the American Heart Association. Circulation. 2014;129(3):399-410.

3. Tsao CW, Lyass A, Enserro D, Larson MG, Ho JE, Kizer JR, et al. Temporal trends in the incidence of and mortality associated with heart failure with preserved and reduced ejection fraction. JACC Heart Fail. 2018;6(8):678-85.

4. Shah SJ, Kitzman DW, Borlaug BA, Heerebeek L, Zile MR, Kass DA, et al. Phenotype-specific treatment of heart failure with preserved ejection fraction: a multiorgan roadmap. Circulation. 2016;134(1):73-90.

5. Yancy CW, Jessup M, Bozkurt B, Butler J, Casey Jr DE, Drazner MH, et al. 2013 ACCF/AHA guideline for the management of heart failure: a report of the American College of Cardiology Foundation/American Heart Association Task Force on Practice Guidelines. J Am Coll Cardiol. 2013;62(16):e147-239.

6. Pieske B, Tschope C, Boer RA, Fraser AG, Anker SD, Donal E, et al. How to diagnose heart failure with preserved ejection fraction: the HFA-PEFF diagnostic algorithm: a consensus recommendation from the Heart Failure Association (HFA) of the European Society of Cardiology (ESC). Eur Heart J. 2019;40(40):3297-3317.

7. Chow SL, Maisel AS, Anand I, Bozkurt B, Boer RA, Felker GM, et al. Role of biomarkers for the prevention, assessment, and management of heart failure: a scientific statement from the American Heart Association. Circulation. 2017;135(22):e1054-91.

\section{Review Criteria}

A search of the PubMed database was performed using the following terms: "interventional therapy heart failure with preserved ejection fraction", "interatrial shunt", "atrial shunting" and "atrial septostomy". Only full-text, peer-reviewed articles published in English were included, predominantly from 2000 to 2020. Bibliographies of the studies identified in the search were also reviewed for additional papers relevant to this topic.

\section{Author Contributions}

Writing of the manuscript: Bhardwaj A, Parikh V, Nair A.

\section{Potential Conflict of Interest}

No potential conflict of interest relevant to this article was reported.

\section{Sources of Funding}

There were no external funding sources for this study.

\section{Study Association}

This study is not associated with any thesis or dissertation work.

8. Lam CS, Rienstra M, Tay WT, Liu LCY, Hummel YM, Meer P, et al. Atrial fibrillation in heart failure with preserved ejection fraction: association with exercise capacity, left ventricular filling pressures, natriuretic peptides, and left atrial volume. JACC Heart Fail. 2017;5(2):92-8.

9. Obokata M, Reddy YNV, Pislaru SV, Melenovsky V, Borlaug BA. Evidence supporting the existence of a distinct obese phenotype of heart failure with preserved ejection fraction. Circulation. 2017;136(1):6-19.

10. Reddy YNV, Carter RE, Obokata M, Redfield MM, Borlaug BA. A simple, evidence-based approach to help guide diagnosis of heart failure with preserved ejection fraction. Circulation. 2018;138(9):861-70.

11. Kim MN, Park SM. Heart failure with preserved ejection fraction: insights from recent clinical researches. Korean J Intern Med. 2020;35(4):1026.

12. Paulus WJ, Tschope C. A novel paradigm for heart failure with preserved ejection fraction: comorbidities drive myocardial dysfunction and remodeling through coronary microvascular endothelial inflammation. J Am Coll Cardiol. 2013;62(4):263-71.

13. Borlaug BA. The pathophysiology of heart failure with preserved ejection fraction. Nat Rev Cardiol. 2014;11(9):507-15.

14. Dorfs S, Zeh W, Hochholzer W, Jander N, Kienzle RP, Pieske B, et al. Pulmonary capillary wedge pressure during exercise and long-term mortality in patients with suspected heart failure with preserved ejection fraction. Eur Heart J. 2014;35(44):3103-12.

15. Rossi A, Gheorghiade M, Triposkiadis F, Solomon SD, Pieske B, Butler J. Left atrium in heart failure with preserved ejection fraction: structure, function, and significance. Circ Heart Fail. 2014;7(6):1042-9. 
16. Melenovsky V, Hwang SJ, Redfield MM, Zakeri R, Lin G, Borlaug BA. Left atrial remodeling and function in advanced heart failure with preserved or reduced ejection fraction. Circ Heart Fail. 2015;8(2):295-303.

17. Gibson DN, Di Biase L, Mohanty P, Patel JD, Bai R, Sanchez J, et al. Stiff left atrial syndrome after catheter ablation for atrial fibrillation: clinical characterization, prevalence, and predictors. Heart Rhythm. 2011;8(9):1364-71.

18. Obokata M, Reddy YNV, Yang JH, Wiley BM, Borlaug BA. Left atrial contracture or failure to dilate. Circ Heart Fail. 2018;11(9):e005163.

19. Reddy YNV, Obokata M, Wiley B, Koepp KE, Jorgenson CC, Egbe A, et al. The haemodynamic basis of lung congestion during exercise in heart failure with preserved ejection fraction. Eur Heart J. 2019;40(45):3721-30.

20. Chandrashekar P, Park JY, Al-Hijji MA, Reddy YNV, Zack C, Reeder GS, et al. Atrial septostomy to treat stiff left atrium syndrome. Circ Heart Fail. 2017;10(7):e004160

21. Nair A, Deswal A. Aldosterone receptor blockade in heart failure with preserved ejection fraction. Heart Fail Clin. 2018;14(4):525-35.

22. Yusuf S, Pfeffer MA, Swedberg K, Granger CB, Held P, McMurray JVM, et al. Effects of candesartan in patients with chronic heart failure and preserved left-ventricular ejection fraction: the CHARM-Preserved Trial. Lancet. 2003;362(9386):777-81.

23. Pitt B, Pfeffer MA, Assmann SF, Boineau R, Anand IS, Clagget B, et al. Spironolactone for heart failure with preserved ejection fraction. N Engl J Med. 2014;370(15):1383-92.

24. Denus S, O'Meara E, Desai AS, Claggett B, Lewis EF, Leclair G, et al. Spironolactone metabolites in TOPCAT - new insights into regional variation. N Engl J Med. 2017;376(17):1690-92.

25. Solomon SD, McMurray JJV, Anand IS, Ge J, Lam CSP, Maggioni AP, et al. Angiotensin-Neprilysin Inhibition in Heart Failure with Preserved Ejection Fraction. N Engl J Med. 2019;381(17):1609-20.

26. McMurray JJV, Jackson AM, Lam CSP, Redfield MM, Anand IS, Ge J, et al. Effects of sacubitril-valsartan versus valsartan in women compared with men with heart failure and preserved ejection fraction: insights from PARAGONHF. Circulation. 2020;141(5):338-51.

27. Gorter TM, Obokata M, Reddy YNV, Melenovsky V, Borlaug BA Exercise unmasks distinct pathophysiologic features in heart failure with preserved ejection fraction and pulmonary vascular disease. Eur Heart J. 2018;39(30):2825-35.

28. Westermann D, Kasner M, Steendijk P, Spillmann F, Riad A, Weitmann K, et al. Role of left ventricular stiffness in heart failure with normal ejection fraction. Circulation. 2008;117(16):2051-60.

29. Hasenfuss G, Hayward C, Burkhoff D, Silvestry FE, Mckenzie S, Gustafsson $\mathrm{F}$, al. A transcatheter intracardiac shunt device for heart failure with preserved ejection fraction (REDUCE LAP-HF): a multicentre, open-label, single-arm, phase 1 trial. Lancet. 2016;387(10025):1298-1304.

30. Kaye D, Shah SJ, Borlaug BA, Gustafsson F, Komtebedde J, Kubo S, et al. Effects of an interatrial shunt on rest and exercise hemodynamics: results of a computer simulation in heart failure. J Card Fail. 2014;20(3):212-21.

31. Guimaraes L, Del Val D, Bergeron S, O'Connor K, Bernier M, Rodes-Cabau J. Interatrial shunting for treating acute and chronic left heart failure. Eur Cardiol. 2020 Apr 27;15:e18.

32. Sondergaard L, Reddy V, Kaye D, Malek F, Walton A, Mates M, et al Transcatheter treatment of heart failure with preserved or mildly reduced ejection fraction using a novel interatrial implant to lower left atrial pressure. Eur J Heart Fail. 2014;16(7):796-801.
33. Del Trigo M, Bergeron S, Bernier M, Amat-Santos IJ, Puri R, CampeloParada F, et al. Unidirectional left-to-right interatrial shunting for treatment of patients with heart failure with reduced ejection fraction: a safety and proof-of-principle cohort study. Lancet. 2016;387(10025):1290-7.

34. Guimaraes L, Lindenfeld J, Sandoval J, Bayés-Genis A, Bernier M, Provencher $\mathrm{S}$, et al. Interatrial shunting for heart failure: current evidence and future perspectives. EuroIntervention. 2019;15(2):164-71.

35. Amat-Santos IJ, Bergeron S, Bernier M, Allende R, Ribeiro HB, Urena M, et al. Left atrial decompression through unidirectional left-to-right interatrial shunt for the treatment of left heart failure: first-in-man experience with the V-Wave device. EuroIntervention. 2015;10(9):1127-31.

36. Paitazoglou C, Ozdemir R, Pfister R, Bergmann MW, Bartunek J, Kilic T, et al. The AFR-PRELIEVE trial: a prospective, non-randomised, pilot study to assess the Atrial Flow Regulator (AFR) in heart failure patients with either preserved or reduced ejection fraction. EuroIntervention. 2019;15(5):403-10.

37. Hasenfuss G, Gustafsson F, Kaye D, Shah SJ, Burkhoff D, Reymond MC, et al. Rationale and design of the reduce elevated left atrial pressure in patients with heart failure (Reduce LAP-HF) trial. J Card Fail. 2015;21(7):594-600.

38. Kaye DM, Hasenfuss G, Neuzil P, Post MC, Doughty R, Trochu JN, et al. One-Year outcomes after transcatheter insertion of an interatrial shunt device for the management of heart failure with preserved ejection fraction. Circ Heart Fail. 2016;9(12):e003662.

39. Kaye DM, Petrie MC, McKenzie S, Hasenfuß G, Malek F, Post M, et al. Impact of an interatrial shunt device on survival and heart failure hospitalization in patients with preserved ejection fraction. ESC Heart Fail. 2019;6(1):62-9.

40. Feldman T, Mauri L, Kahwash R, Litwin S, Ricciardi MJ, Harst P, et al. Transcatheter interatrial shunt device for the treatment of heart failure with preserved ejection fraction (REDUCE LAP-HF I [Reduce Elevated Left Atrial Pressure in Patients With Heart Failure]): a phase 2, randomized, sham-controlled trial. Circulation. 2018;137(4):364-75.

41. Shah SJ, Feldman T, Ricciardi MJ, Kahwash R, Lilly S, Litwin S, et al. One-Year Safety and clinical outcomes of a transcatheter interatrial shunt device for the treatment of heart failure with preserved ejection fraction in the reduce elevated left atrial pressure in patients with heart failure (REDUCE LAP-HF I) trial: a randomized clinical trial. JAMA Cardiol. 2018;3(10):968-77.

42. Obokata M, Reddy YNV, Shah SJ, Kaye DM, Gustafsson F, Hasenfuß $\mathrm{G}$, et al. Effects of interatrial shunt on pulmonary vascular function in heart failure with preserved ejection fraction. J Am Coll Cardiol. 2019;74(21):2539-50

43. Rodes-Cabau J, Bernier M, Amat-Santos IJ, Gal TB, Nombela-Franco L. Blanco BG, et al. Interatrial shunting for heart failure: early and late results from the first-in-human experience with the V-Wave System. JACC Cardiovasc Interv. 2018;11(22):2300-10.

44. Guimaraes L, Bergeron S, Bernier M, Rodriguez-Gabella T, Val DD, Pibarot $\mathrm{P}$, et al. Interatrial shunt with the second-generation V-Wave system for patients with advanced chronic heart failure. EuroIntervention. 2020;15(16):1426-8.

45. Zeitler EP, Abraham WT. Novel devices in heart failure: BAT, atrial shunts, and phrenic nerve stimulation. JACC Heart Fail. 2020;8(4):251-64.

46. Nichols GA, Reynolds K, Kimes TM, Rosales AG, Chan WW. Comparison of risk of re-hospitalization, all-cause mortality, and medical care resource utilization in patients with heart failure and preserved versus reduced ejection fraction. Am J Cardiol. 2015;116(7):1088-92. 